\title{
Impact of Obesity on the Rate of Recurrent Spontaneous Preterm Birth in Women Treated with 17-alpha Hydroxyprogesterone Caproate
}

\author{
Alisse Hauspurg, MD ${ }^{1}$ Lara S. Lemon, PharmD ${ }^{2,3}$ Allison E. Serra, MD, MPH ${ }^{1} \quad$ Shringi Sharma, $\mathrm{PhD}^{3}$ \\ Raman Venkataramanan, $\mathrm{PhD}^{3,4}$ Steve N. Caritis, MD ${ }^{1}$
}

${ }^{1}$ Division of Maternal-Fetal Medicine, Department of Obstetrics, Gynecology, and Reproductive Sciences, Magee-Womens Hospital of UPMC, Pittsburgh, Pennsylvania

Address for correspondence Alisse Hauspurg, MD, 300 Halket Street,

2 Department of Epidemiology, Graduate School of Public Health, University of Pittsburgh, Pittsburgh, Pennsylvania

${ }^{3}$ Department of Pharmaceutical Science, School of Pharmacy, University of Pittsburgh, Pittsburgh, Pennsylvania

${ }^{4}$ Department of Pathology, School of Medicine, University of Pittsburgh, Pittsburgh, Pennsylvania

Am J Perinatol 2018;35:809-814.

\begin{abstract}
Keywords

- 17- $\alpha$ hydroxyprogesterone caproate

- BMI

- obesity

- preterm birth

- recurrent preterm birth

- spontaneous preterm birth

Objective We sought to determine if the rate of recurrent spontaneous preterm birth (PTB) in women treated with $17-\alpha$ hydroxyprogesterone caproate (17-OHPC) is modified by maternal body mass index (BMI).

Study Design We performed a secondary analysis of the Maternal-Fetal Medicine Units Network omega-3 fatty acid supplementation to prevent recurrent PTB randomized controlled trial. All women received 17-OHPC.

Results A total of 708 women were included. Rates of spontaneous PTB did not vary significantly by BMI category. With stratification by obesity class and gestational age at delivery, the unadjusted risk for PTB using earlier gestational cutoffs $(<35,32$, and 28 weeks) demonstrated an association between preterm delivery and increasing severity of obesity. With adjustment for potential confounders, there was no statistically significant relationship between BMI and spontaneous PTB.

Conclusion We demonstrated that the risk of PTB in women receiving $250 \mathrm{mg}$ 17-OHPC is not dependent on maternal BMI after adjustment for confounding variables. Pharmacokinetic studies have demonstrated a wide variation in plasma concentration of 17-OHPC across the population with likely considerable overlap in plasma concentrations among the obese and nonobese population. Further studies are needed to evaluate the impact of BMI on efficacy of 17-OHPC prior to any dose adjustment in this population.
\end{abstract}

In 2014, the preterm birth (PTB) rate in the United States was $9.6 \%{ }^{1}$ Weekly administration of intramuscular $17-\alpha$ hydroxyprogesterone caproate (17-OHPC) represents one of the few effective interventions to reduce recurrence of preterm delivery, with a reduction in the risk of recurrent preterm delivery by approximately one-third. ${ }^{2}$ The $250 \mathrm{mg}$ weekly dosage of 17 OHPC used in the Meis et al trial and widely adopted into clinical practice was extrapolated from prior trials and expert opinion. ${ }^{2}$ Currently, no data exist to guide optimal dosing of 17-OHPC or therapeutic plasma concentrations necessary to minimize rates received

June 21, 2017

accepted after revision

November 29, 2017

published online

January 2, 2018
Copyright $\odot 2018$ by Thieme Medical Publishers, Inc., 333 Seventh Avenue, New York, NY 10001, USA. Tel: +1(212) 584-4662.
DOI https://doi.org/ 10.1055/s-0037-1617453. ISSN 0735-1631. 
of PTB. Recent pharmacokinetic simulations from our group suggest that plasma 17-OHPC levels are impacted by maternal body mass index (BMI). Based on a secondary analysis, we have also shown that women with plasma 17-OHPC levels below a threshold concentration experience higher rates of PTB. ${ }^{3,4} \mathrm{We}$ have reported that the volume of distribution for 17-OHPC is more in obese than nonobese women and since 17-OHPC is highly lipophilic, the question is raised whether 17-OHPC is less effective in women with a higher BMI or body weight. However, prior studies attempting to answer this question have provided conflicting results. ${ }^{5-8}$ To address this question, we utilized two datasets. First, we performed a secondary analysis of the Eunice Kennedy Shriver National Institute of Child Health and Human Development Maternal-Fetal Medicine Units Network (NICHD MFMU) omega-3 randomized controlled trial by Harper et al. ${ }^{9}$ In addition, we utilized data from the Obstetrical-Fetal Pharmacology Research Units (OPRU) Network study describing the pharmacokinetics of 17-OHPC in women with singleton gestation. ${ }^{10,11}$ Our hypothesis was that the rate of recurrent spontaneous PTB in women treated with 17-OHPC is modified by maternal BMI.

\section{Materials and Methods}

This is a secondary analysis of data collected in a randomized double-masked placebo controlled trial conducted by the NICHD MFMU Network. The primary study was conducted at 13 centers between January 2005 and October 2006. The original study design and population have been previously described. ${ }^{9}$ Approval from the Institutional Review Board was received at each institution for the original study. All women who participated in the study provided written informed consent. Briefly, women with a current singleton pregnancy between 16 and $21^{6 / 7}$ weeks who were at increased risk for PTB (history of one or more documented singleton spontaneous preterm deliveries between $20^{0 / 7}$ and $36^{6 / 7}$ weeks of gestation) were recruited. Exclusion criteria were women with excess fish oil supplement intake, allergy to fish, anticoagulation therapy, hypertension, White's classification D or higher diabetes, drug or alcohol abuse, seizure disorder, uncontrolled thyroid disease, clotting disorder, current or planned cerclage, or a plan to deliver elsewhere, plan to deliver before 37 weeks of gestation, or evidence of a major fetal anomaly.

Consenting eligible women received an injection of $250 \mathrm{mg}$ of 17-OHPC and a 7-day supply of placebo capsules. Those who either did not return after 5 days and before $21^{6 / 7}$ weeks of gestation or had taken less than half of the placebo capsules were not allowed to participate. Women passing the run-in phase were randomly assigned to receive either a daily supplement containing $1,200 \mathrm{mg}$ of eicosapentaenoic acid (20:5n-3) and $800 \mathrm{mg}$ of docosahexaenoic acid (22:6n-3), for a total of 2,000 $\mathrm{mg}$ of omega-3 long-chain polyunsaturated fatty acids, divided into four capsules, or matching placebo capsules, which contained only a minute amount of inert mineral oil. All women received weekly injections of 17OHPC (250 mg) as described in the trial by Meis et al. ${ }^{2}$ Study drug and 17-OHPC injections were continued until delivery or $36^{6 / 7}$ weeks of gestation, whichever occurred first. This trial found no benefit of omega-3 long-chain polyunsaturated fatty acid supplementation in reducing PTB. Thus, for this study, we included women in both the treatment and placebo groups.

As we did not have access to plasma concentrations of 17-OHPC in this cohort, we also present data from a multicenter OPRU Network study evaluating the pharmacokinetics of 17-OHPC to assess the impact of BMI on plasma levels of 17-OHPC. The original study design and population have been described previously. ${ }^{11}$ This study included 61 women from four centers who received 17-OHPC based on a history of at least one prior spontaneous PTB and participated in a pharmacokinetic study between $31^{0 / 7}$ and $34^{6 / 7}$ weeks of gestation. At each pharmacokinetic study, venous blood was obtained daily for 7 days prior to the next injection of 17-OHPC but after steady state had been reached. Women were included if they participated in the pharmacokinetic study between $31^{0 / 7}$ and $34^{6 / 7}$ weeks and had an enrollment BMI recorded $(n=43)$. In this study, we compared trough plasma concentrations of 17-OHPC obtained immediately prior to the next injection to pre-pregnancy BMI.

For the omega- 3 cohort, a total of 3,755 women were screened; 994 participated in the run-in phase, and 852 of these passing the compliance testing were randomly assigned to treatment. Of those assigned to treatment, 434 were randomly assigned to the omega-3 supplement arm and 418 to the placebo arm. All women received 17-OHPC. The primary outcome was available for all 852 women. In this secondary analysis, women were excluded due to poor compliance (less than 50\% compliance with weekly injections), a cervical length less than $2.5 \mathrm{~cm}$, underweight BMI $\left(<18.5 \mathrm{~kg} / \mathrm{m}^{2}\right)$, missing information on maternal BMI or for a medically indicated PTB (-Fig. 1).

For this analysis, data were analyzed with subjects grouped according to BMI prior to pregnancy: normal (BMI: 18.5-24.9), overweight (BMI: 25.0-29.9), obese class I (BMI: 30.09-34.9), class II (BMI: 35.0-39.9) and class III (BMI: 40 and above). Due to limited sample size in classes II and III obese category, we also performed analyses after consolidating the three obesity classes into one group "obese" (BMI: 30 and above). The primary outcome was spontaneous preterm delivery prior to 37 weeks. Secondary outcomes included delivery before 32 weeks and before 28 weeks. Statistical analysis was conducted using STATA software, version 14 (StataCorp, College Station, TX). Continuous variables were compared using Student's t-tests and Wilcoxon-Mann-Whitney's tests as appropriate. Categorical variables were analyzed using chi-square or Fisher's exact test, where appropriate. Multivariable analysis included log binomial regression to evaluate the independent association between maternal body habitus (as a discrete variable: obese/ overweight vs. normal, and as a continuous variable: increasing BMI) and spontaneous preterm delivery, a relatively common outcome. Adjustment covariates were chosen a priori based on previous literature and included age $>30$ years, marital status, years of education, smoking, $>1$ previous PTB, earliest prior PTB $<28$ weeks. Results were presented as adjusted risk ratios with corresponding 95\% confidence intervals, and a $p$-value $<0.05$ was considered statistically significant. No adjustments were made for multiple comparisons. 


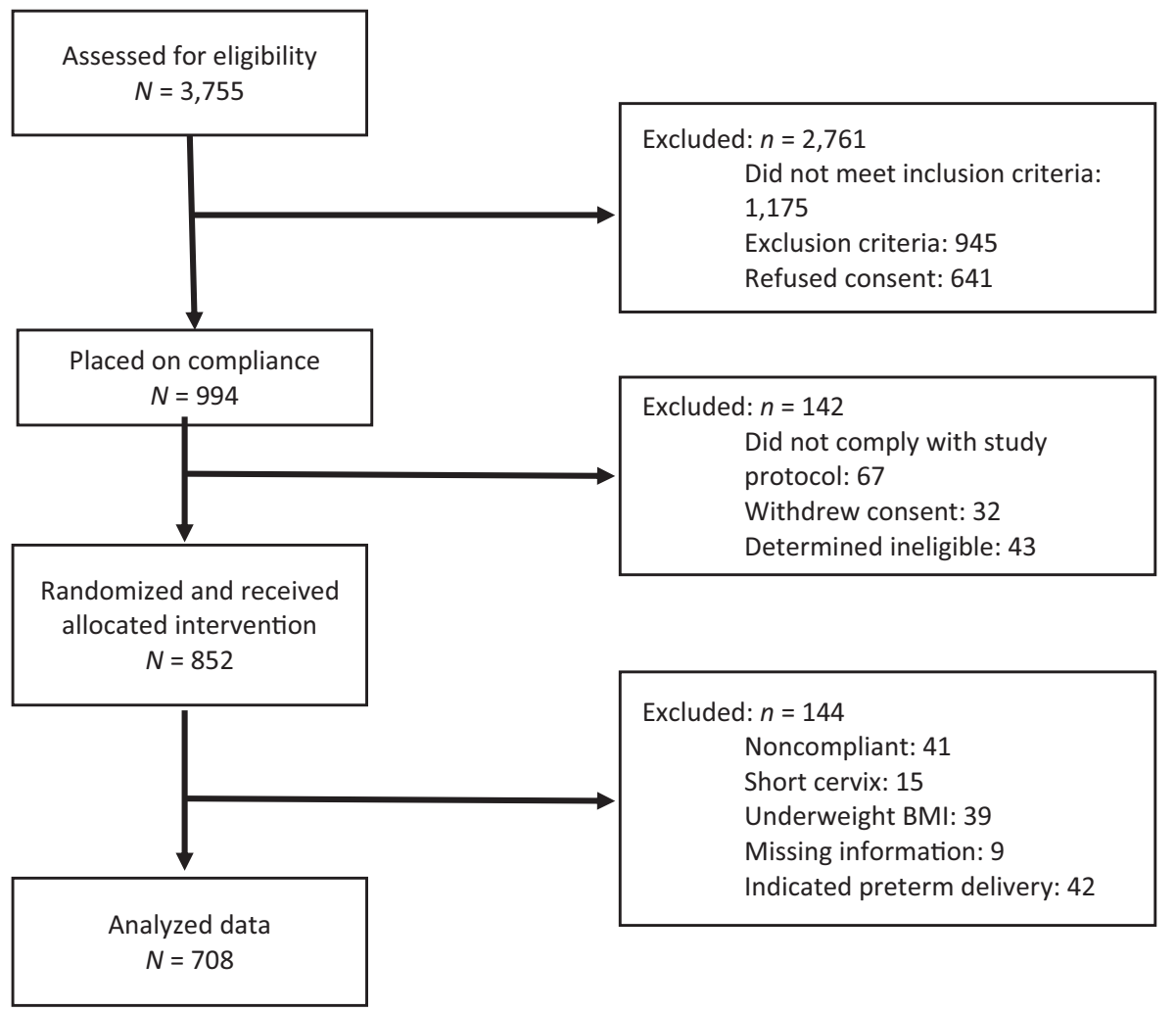

Fig. 1 Screening, randomization, and inclusion of study participants.

\section{Results}

A total of 708 women were included in the final cohort. Demographics of our final analytic sample are shown in -Table 1. Overweight and obese women in our cohort were more likely to be non-Hispanic black, had fewer years of schooling, had a higher parity, and were more likely to have preeclampsia or gestational hypertension in the current pregnancy than normal weight women. There was no difference in average gestational age at delivery.

Overall, the rates of spontaneous PTB at less than 37 weeks did not vary significantly by BMI category (- Table 2, - Fig. 2). With stratification by obesity class and various gestational age cutoffs ( $<35,32$, and 28 weeks), there were still no significant relationships between BMI category and the rate of PTB. Women with more severe obesity (BMI $\geq 35 \mathrm{~kg} / \mathrm{m}^{2}$ ) had significantly increased rates of spontaneous PTB before 28 and 32 weeks compared with normal weight women. About $9 \%$ ( 8 out of 91 ) of women with a BMI $\geq 35 \mathrm{~kg} / \mathrm{m}^{2}$ delivered prior to 28 weeks, compared with $2.8 \%$ of normal weight women $(p=0.01)$. Similarly, $14.3 \%$ (13 out of 91$)$ of women with a $\mathrm{BMI} \geq 35 \mathrm{~kg} / \mathrm{m}^{2}$ delivered prior to 32 weeks compared with $7.4 \%$ (25 out of 326 ) of normal weight women ( $p=0.04$ ).

When the three obesity categories were collapsed into one category for comparison of normal women to obese women, spontaneous PTB occurred in $37.4 \%$ of normal weight women compared with $31.5 \%$ of overweight women and $34.3 \%$ of obese women. Although not statistically significant, there was a trend toward earlier PTB in obese women; $2.8 \%$ of normal weight women and 5.9\% of obese women delivered prior to 28 weeks ( $p=0.073$ ) and $7.4 \%$ of normal weight women and $12.3 \%$ of obese women delivered prior to 32 weeks ( $p=0.058$ ).

Logistic regression analysis was performed to further characterize the relationship between early preterm delivery and increasing maternal BMI. The unadjusted risk of recurrent spontaneous PTB prior to 28 and 32 weeks in women with class III obesity was $3.62(1.17-11.23)$ and 2.04 (0.894.69), respectively. When we further adjusted for race, older maternal age, marital status, years of education, smoking, more than one prior PTB and early gestational age of qualifying PTB, the risk ratios showed no statistically significant relationship between BMI and spontaneous PTB at earlier gestational ages (- Table $\mathbf{3}$ ). This finding was similar whether BMI was assessed as a continuous variable or categorically and at later gestational age cutoffs ( 35 and 37 weeks).

To assess the relationship between 17-OHPC concentration and maternal BMI, we used available data from a smaller cohort study through the OPRU as plasma levels from the omega-3 cohort were not available for this analysis. ${ }^{11}$ Demographic characteristics of this cohort have been described previously. ${ }^{11}$ Briefly, these women had a mean of 1.5 prior PTBs. Racial distribution was similar to omega- 3 cohort with $49 \%$ Caucasian, 21\% African American, 26\% Hispanic, and 3\% other. Mean BMI in this cohort was $29.0 \mathrm{~kg} / \mathrm{m}^{2}$. As shown in - Fig. 3, plasma trough concentrations obtained between $31^{0 / 7}$ and $34^{6 / 7}$ weeks' gestation varied considerably across the population and were not strongly associated with maternal BMI $\left(r^{2}=0.17\right)$. BMI accounts for only $17 \%$ of the variance in plasma $17-\mathrm{OHPC}$ concentration. 
812 Impact of Obesity on the Rate of Recurrent Spontaneous PTB in Women Treated with 17-OHPC Hauspurg et al.

Table 1 Demographics of women in omega-3 cohort included in analysis $(n=708)$

\begin{tabular}{|c|c|c|c|}
\hline & $\begin{array}{l}\text { Normal BMI } \\
(\text { BMI 19.5- }<25) \\
n=326 \\
N(\%) \\
\end{array}$ & $\begin{array}{l}\text { Overweight and obese } \\
\text { (BMI } \geq 25) \\
n=382 \\
N(\%) \\
\end{array}$ & $p$-Value \\
\hline \multicolumn{4}{|l|}{ Race } \\
\hline Non-Hispanic white & $198(60.7)$ & $156(40.8)$ & \multirow[t]{4}{*}{$<0.001$} \\
\hline Non-Hispanic black & $72(22.1)$ & $162(42.4)$ & \\
\hline Hispanic & $44(13.5)$ & $56(14.7)$ & \\
\hline Other & $12(3.7)$ & $8(2.1)$ & \\
\hline Mother's age $^{a}$ & $27.8 \pm 5.5$ & $27.8 \pm 5.5$ & 0.904 \\
\hline \multicolumn{4}{|l|}{ Years of maternal schooling } \\
\hline$\leq 6 \mathrm{y}$ & $1(0.3)$ & $4(1.1)$ & \multirow[t]{3}{*}{0.008} \\
\hline $7-12$ y & $125(38.3)$ & $186(48.7)$ & \\
\hline$\geq 13$ y & $200(61.4)$ & $192(50.3)$ & \\
\hline \multicolumn{4}{|l|}{ Marital status } \\
\hline Married & $243(74.5)$ & $255(66.7)$ & \multirow[t]{3}{*}{0.053} \\
\hline Divorced/widowed & $10(3.1)$ & $21(5.5)$ & \\
\hline Never married & $73(22.4)$ & $106(27.8)$ & \\
\hline \multicolumn{4}{|l|}{ Smoking status prerandomization } \\
\hline Smoker & $40(12.3)$ & $63(16.5)$ & \multirow[t]{2}{*}{0.112} \\
\hline Nonsmoker & $286(87.7)$ & $319(83.5)$ & \\
\hline \multicolumn{4}{|l|}{ Parity } \\
\hline 1 previous pregnancy & $170(52.2)$ & $164(42.9)$ & \multirow[t]{3}{*}{0.021} \\
\hline 2 previous pregnancies & $93(28.5)$ & $115(30.1)$ & \\
\hline 3 or more pregnancies & $63(19.3)$ & $103(27.0)$ & \\
\hline Diabetes at baseline & $5(1.5)$ & $6(1.6)$ & 0.968 \\
\hline Average gestational age at delivery $(w k)^{a}$ & $36.8 \pm 3.4$ & $36.7 \pm 4.1$ & 0.232 \\
\hline Preeclampsia or gestational hypertension & $4(1.2)$ & $19(5.0)$ & 0.005 \\
\hline
\end{tabular}

Abbreviation: BMI, body mass index.

${ }^{\text {a Mean }} \pm$ standard deviation.

Table 2 Rate of spontaneous preterm birth by BMI category and gestation at delivery ${ }^{\mathrm{a}, \mathrm{b}}$

\begin{tabular}{|l|l|l|l|l|l|}
\hline & $\begin{array}{l}\text { Normal weight } \\
n=326 \\
N(\%)\end{array}$ & $\begin{array}{l}\text { Overweight } \\
n=178 \\
N(\%)\end{array}$ & $\begin{array}{l}\text { Class I obese } \\
n=113 \\
N(\%)\end{array}$ & $\begin{array}{l}\text { Class II obese } \\
n=51 \\
N(\%)\end{array}$ & $\begin{array}{l}\text { Class III obese } \\
n=40 \\
N(\%)\end{array}$ \\
\hline Delivery $<37 \mathrm{wk}$ & $122(37.4)$ & $56(31.5)$ & $37(32.7)$ & $18(35.3)$ & $15(37.5)$ \\
\hline Delivery $<35 \mathrm{wk}$ & $51(15.6)$ & $28(15.7)$ & $20(17.7)$ & $10(19.6)$ & $8(20.0)$ \\
\hline Delivery $<32 \mathrm{wk}$ & $25(7.4)$ & $14(7.9)$ & $12(10.6)$ & $7(13.7)$ & $6(15.0)$ \\
\hline Delivery $<28 \mathrm{wk}$ & $9(2.8)$ & $7(3.9)$ & $4(3.5)$ & $4(7.8)$ & $4(10.0)$ \\
\hline
\end{tabular}

Abbreviation: BMI, body mass index.

aPre-pregnancy BMI defined as normal weight $\left(18.5-<25 \mathrm{~kg} / \mathrm{m}^{2}\right)$, overweight $\left(25-<30 \mathrm{~kg} / \mathrm{m}^{2}\right)$, class 1 obesity $\left(30-<35 \mathrm{~kg} / \mathrm{m}^{2}\right)$, class $2 \mathrm{obesity}$ $\left(35-<40 \mathrm{~kg} / \mathrm{m}^{2}\right)$, and class 3 obesity $\left(\geq 40 \mathrm{~kg} / \mathrm{m}^{2}\right)$.

${ }^{\mathrm{b}}$ There are no significant differences between subgroups.

\section{Discussion}

We have demonstrated that the risk of PTB in women receiving $250 \mathrm{mg}$ 17-OHPC is not associated with maternal BMI. Despite controlling for appropriate confounders, prior studies have shown conflicting results concerning the relationship of maternal weight and BMI to outcomes with 17-OHPC for prevention of recurrent PTB. A retrospective cohort study ${ }^{5}$ of 390 women treated with 17-OHPC for a history of prior spontaneous PTB found that recurrent PTB before 32 weeks was significantly 


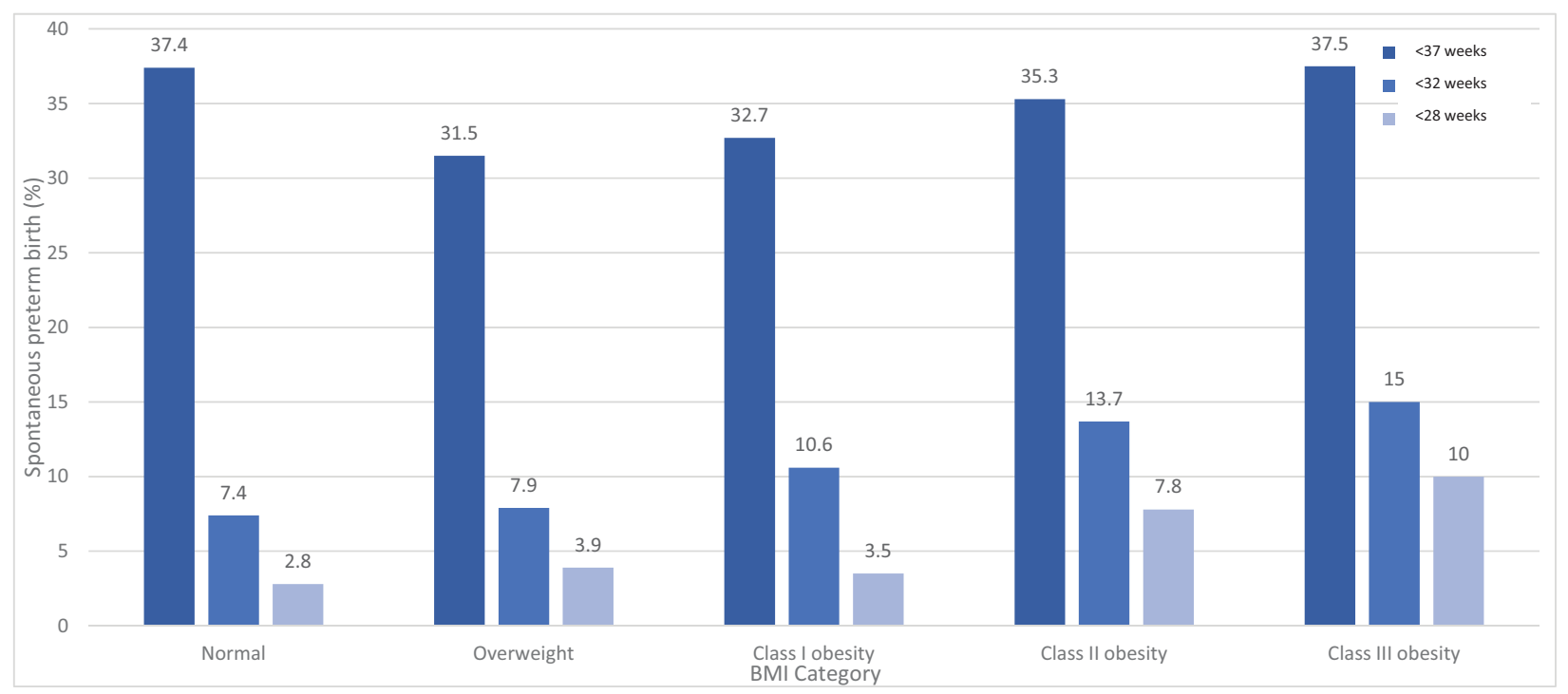

Fig. 2 Rate of spontaneous preterm birth prior to 37, 32, and 28 weeks' gestational age by BMI category. Pre-pregnancy BMI defined as normal weight $\left(18.5-<25 \mathrm{~kg} / \mathrm{m}^{2}\right)$, overweight $\left(25-<30 \mathrm{~kg} / \mathrm{m}^{2}\right)$, class 1 obesity $\left(30-<35 \mathrm{~kg} / \mathrm{m}^{2}\right)$, class 2 obesity $\left(35-<40 \mathrm{~kg} / \mathrm{m}^{2}\right)$, and class 3 obesity $\left(\geq 40 \mathrm{~kg} / \mathrm{m}^{2}\right)$. BMI, body mass index.

Table 3 RRs of spontaneous preterm delivery prior to 28 and 32 weeks associated with BMI

\begin{tabular}{|l|l|l|l|l|}
\hline & \multicolumn{2}{|l|}{ Delivery before 32 wk } & \multicolumn{2}{l|}{ Delivery before 28 wk } \\
\cline { 2 - 5 } & Unadjusted RR (95\% Cl) & Adjusted $^{\mathrm{a}}$ RR (95\% CI) & Unadjusted RR (95\% CI) & Adjusted $^{\mathrm{a}}$ RR (95\% CI) \\
\hline BMI continuous & $1.04(1.01-1.07)$ & $1.02(0.99-1.05)$ & $1.05(1.01-1.10)$ & $1.02(0.98-1.07)$ \\
\hline Normal weight & Reference & Reference & Reference & Reference \\
\hline Overweight & $1.07(0.57-2.01)$ & $1.04(0.55-1.98)$ & $1.42(0.54-3.76)$ & $1.41(0.56-3.59)$ \\
\hline Class 1 obesity & $1.44(0.75-2.79)$ & $1.37(0.70-2.68)$ & $1.28(0.40-4.09)$ & $1.26(0.38-4.16)$ \\
\hline Class 2 obesity & $1.86(0.85-4.10)$ & $1.48(0.67-3.28)$ & $2.84(0.91-8.89)$ & $1.96(0.63-6.13)$ \\
\hline Class 3 obesity & $2.04(0.89-4.69)$ & $1.59(0.71-3.56)$ & $3.62(1.17-11.23)$ & $1.89(0.60-5.93)$ \\
\hline
\end{tabular}

Abbreviation: $\mathrm{BMI}$, body mass index; $\mathrm{Cl}$, confidence interval; $\mathrm{RR}$, relative risk.

${ }^{a}$ Adjusted for race, age $>30$ years, marital status, years of education, smoking, $>1$ previous preterm birth, earliest prior preterm birth $<28$ weeks.

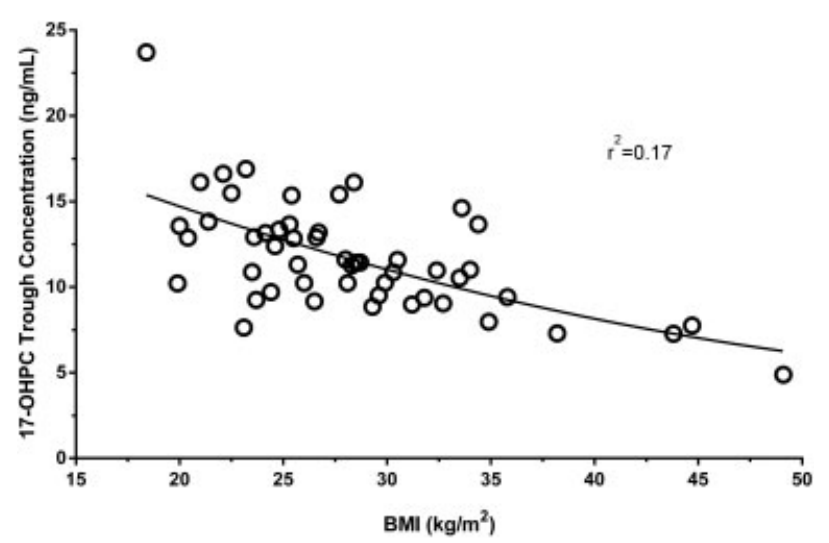

Fig. 3 Relationship of 17-OHPC trough concentration and BMI. BMI, body mass index; 17-OHPC, 17- $\alpha$ hydroxyprogesterone caproate.

more common in overweight and obese women compared with women with a BMI less than $25 \mathrm{~kg} / \mathrm{m}^{2}$ even after adjustment for age, race, smoking status, short cervical length, and gestational age of patient's earliest PTB. This study was limited by a smaller overall cohort size. Heyborne et $\mathrm{al}^{6}$ recently performed a secondary analysis of the Meis et al trial, which included 443 women and concluded that $250 \mathrm{mg}$ of intramuscular 170HP-C weekly is ineffective in prevention of recurrent PTB in obese women with BMI $>30 \mathrm{~kg} / \mathrm{m}^{2}$ or weight $>165$ pounds. The authors called for urgent consideration of adjusted-dose 17-OHPC studies. A large prospective cohort study $^{8}$ that included 606 women with a history of prior PTB who were receiving $17-\mathrm{OHPC}$ found no significant difference in the incidence of recurrent preterm delivery (at less than 35,32 , or 24 weeks) between BMI groups. The rates of preterm delivery across various gestational age and BMI categories in this study were consistent with our findings. The largest retrospective cohort study ${ }^{7}$ addressing this question included 6,253 women with a history of a prior PTB treated with 17OHPC and actually found a decrease in the rate of recurrent spontaneous PTB with increasing BMI even after controlling for race and prior PTB earlier than 28 weeks.

To our knowledge, our study is the largest secondary analysis of a prospective cohort study examining the relationship 
of maternal BMI to spontaneous PTB rates in women treated with 17-OHPC. While our analysis does not provide the anticipated clarity to the conflicting evidence reported in the literature, it does support the need for further investigation with larger pharmacologic studies. Clearly, previous studies and our current analysis are lacking critical information concerning plasma levels of 17-OHPC. Prior pharmacokinetic analyses performed by our group in both singletons and twins likely prompted the question of whether obese women require a higher dose of $17-\mathrm{OHPC} .^{3,4}$ In one study ${ }^{10}$ from the OPRU, we utilized a cohort of 61 women sampled throughout pregnancy to gain more insight into those factors that affect 17-OHPC plasma concentrations. We demonstrated that obese women have higher volumes of distribution and higher clearance rates ( $\sim 17 \%$ for each) than normal weight women. Conceivably, this relationship likely leads to a decrease in plasma concentration. As we did not have this plasma information in our larger omega-3 cohort available, we utilized available plasma data from this smaller OPRU pharmacokinetic study to demonstrate that plasma levels vary widely across maternal BMI categories. Based on this small cohort, there does not appear to be a strong association between maternal BMI and plasma 17-OHPC concentration. However, there was a wide variation in plasma concentrations of 17-OHPC across the study population, with values ranging from 3.7 to $56 \mathrm{ng} / \mathrm{mL}$ in women receiving the same $250 \mathrm{mg}$ dose of $17-\mathrm{OHPC}$. With this wide variation in plasma levels, it is conceivable that there is considerable overlap in plasma concentrations among the obese and nonobese population. Of greater concern is the percentage of women who achieve therapeutic concentration, and whether this is modified by BMI. In a secondary analysis of the same cohort utilized in the current study, ${ }^{3}$ we reported that plasma concentrations in the lowest quartile of plasma 17-OHPC concentrations had a higher rate of PTB than did women in the three higher quartiles. This relationship between 17-OHPC concentrations and the rate of PTB remained significant after adjustment for maternal BMI suggesting that obese women were not overrepresented in the lowest quartile of concentration.

The picture is further clouded by the dynamic state of pregnancy with individual variability in body weight, protein binding, hormone levels, and drug metabolizing enzyme activity, all of which could affect 17-OHPC pharmacokinetics. All prior studies, ours included, did not account for gestational weight gain, which likely has at least a modest effect on this relationship. ${ }^{12}$ Overall, the literature on this subject matter is conflicting, confusing, and quite limited by small sample sizes. As the number of obese pregnant women in the United States continues to increase, we feel the most effective approach to address this issue is through a prospective pharmacologic study with a large sample size to provide the granularity needed to specifically identify which maternal characteristics that impact plasma 17-OHPC concentrations.

While the preponderance of the pharmacologic evidence suggests that maternal weight and BMI impact plasma levels and also that plasma levels impact efficacy of the drug, without critical information regarding plasma levels in our cohort, our ability to interpret our results is limited. With the current available evidence, a recommendation for dose adjustment of $170 H P C$ in obese pregnant women would be premature.

\section{Funding}

This project was supported by the Obstetric-Fetal Pharmacology Research Centers (OPRC) Grant \#HD047905 and a Ruth Kirschstein T-32 Training Grant \#HD071859.

\section{Conflict of Interest}

None.

\section{References}

1 Hamilton BE, Martin JA, Osterman MJ, Curtin SC, Matthews TJ. Births: final data for 2014. Natl Vital Stat Rep 2015;64(12):1-64

2 Meis PJ, Klebanoff M, Thom E, et al; National Institute of Child Health and Human Development Maternal-Fetal Medicine Units Network. Prevention of recurrent preterm delivery by 17 alpha-hydroxyprogesterone caproate. N Engl J Med 2003;348(24):2379-2385

3 Caritis SN, Venkataramanan R, Thom E, et al; Eunice Kennedy Shriver National Institute of Child Health and Human Development Maternal-Fetal Medicine Units Network and Obstetric-Fetal Pharmacology Research Units Network. Relationship between 17-alpha hydroxyprogesterone caproate concentration and spontaneous preterm birth. Am J Obstet Gynecol 2014;210(02):128.e1-128.e6

4 Caritis SN, Sharma S, Venkataramanan R, et al; Eunice Kennedy Shriver National Institute of Child Health and Human Development Maternal-Fetal Medicine Units Network. Pharmacokinetics of 17-hydroxyprogesterone caproate in multifetal gestation. Am J Obstet Gynecol 2011;205(01):40.e1-40.e8

5 Co AL, Walker HC, Hade EM, Iams JD. Relation of body mass index to frequency of recurrent preterm birth in women treated with 17-alpha hydroxyprogesterone caproate. Am J Obstet Gynecol 2015;213(02):233.e1-233.e5

6 Heyborne KD, Allshouse AA, Carey JC. Does 17-alpha hydroxyprogesterone caproate prevent recurrent preterm birth in obese women? Am J Obstet Gynecol 2015;213(06):844.e1-844.e6

7 Timofeev J, Feghali M, Boyle A, Istwan N, Rhea D, Driggers RW. Rates of recurrent preterm birth by maternal body habitus in women receiving $17 \alpha$-hydroxyprogesterone caproate. J Matern Fetal Neonatal Med 2013;26(09):881-884

8 Ventolini G, Duke J, Po W, et al. The impact of maternal body mass on the effectiveness of 17 alpha-hydroxyprogesterone caproate. J Reprod Med 2008;53(09):667-671

9 Harper M, Thom E, Klebanoff MA, et al; Eunice Kennedy Shriver National Institute of Child Health and Human Development Maternal-Fetal Medicine Units Network. Omega-3 fatty acid supplementation to prevent recurrent preterm birth: a randomized controlled trial. Obstet Gynecol 2010;115(2 Pt 1):234-242

10 Sharma S, Caritis S, Hankins G, et al. Population pharmacokinetics of $17 \alpha$-hydroxyprogesterone caproate in singleton gestation. $\mathrm{Br} \mathrm{J}$ Clin Pharmacol 2016;82(04):1084-1093

11 Caritis SN, Sharma S, Venkataramanan R, et al; Eunice Kennedy Shriver National Institute of Child Health and Human Development Obstetrical-Fetal Pharmacology Research Units Network. Pharmacology and placental transport of 17-hydroxyprogesterone caproate in singleton gestation. Am J Obstet Gynecol 2012; 207(05):398.e1-398.e8

12 Faucher MA, Hastings-Tolsma M, Song JJ, Willoughby DS, Bader SG. Gestational weight gain and preterm birth in obese women: a systematic review and meta-analysis. BJOG 2016;123(02):199-206 